\title{
Histological Analysis of the Tibial Anterior Cruciate Ligament Insertion
}

\author{
Rainer Siebold ${ }^{1,3}$, Shinya Oka ${ }^{1,2}$, Ulrike Traut ${ }^{3}$, Peter Schuhmacher ${ }^{1}$, Joachim Kirsch ${ }^{3}$ \\ 1 HKF - Center for Hip-Knee-Foot Surgery, ATOS Clinic, Heidelberg, Germany \\ 2 Department of Orthopaedic Surgery, Kobe University Graduate School of Medicine, Japan \\ 3 Institute for Anatomy and Cell Biology, Ruprecht-Karls University, INF, Heidelberg, Germany
}

Objective: To describe the morphology of the tibial ACL insertion by histological assessment in the sagittal plane. Methods: For histology the native (undissected) tibial ACL insertion of 6 fresh-frozen cadaveric knees was cut into 4 sagittal sections parallel to the long axis of the medial tibial spine. The slices were stained with hematoxylin and eosin, Safranin O and Russell-Movat pentachrome. All slices were digitalized and analyzed at a magnification of $\times 20$.

Results: From medial to lateral the anterior-posterior lengths of the ACL insertion were an average of 10.2, 9.3, 7.6 and $5.8 \mathrm{~mm}$. The anterior margin of the tibial $A C L$ insertion raised from an anterior ridge. The most medial $A C L$ fibers rose along with a peak of the anterior part of the medial tibial spine in which the direct insertion was adjacent to the articular cartilage. Parts of the bony insertions of the anterior and posterior horns of the lateral meniscus were in close contact to the lateral ACL insertion. A small fat pad was located just posterior to the tibial ACL insertion. There were no central or posterolateral inserting $A C L$ fibers in the area intercondylaris anterior.

Conclusion: The functional intraligamentous midsubstance ACL fibers arose from the most posterior part of its bony tibial insertion in a flat and "C-shape" way. The anterior border of this functional ACL started from a bony 'anterior ridge' and the medial border was along with a peak of the medial tibial spine.

The Orthopaedic Journal of Sports Medicine, 5(5)(suppl 5)

DOI: $10.1177 / 2325967117 S 00179$

(C)The Author(s) 2017 